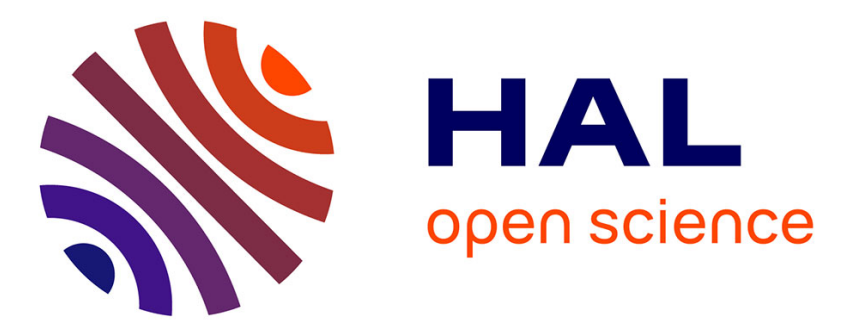

\title{
Synchronous electric charge and induced current extraction (SECICE): a unified nonlinear technique combining piezoelectric and electromagnetic harvesting
}

Giulia Lombardi, Mickaël Null Lallart

\section{- To cite this version:}

Giulia Lombardi, Mickaël Null Lallart. Synchronous electric charge and induced current extraction (SECICE): a unified nonlinear technique combining piezoelectric and electromagnetic harvesting. Smart Materials and Structures, 2021, 30 (2), pp.025029. 10.1088/1361-665X/abd346 . hal-03130996

\author{
HAL Id: hal-03130996 \\ https://hal.science/hal-03130996
}

Submitted on 4 Feb 2021

HAL is a multi-disciplinary open access archive for the deposit and dissemination of scientific research documents, whether they are published or not. The documents may come from teaching and research institutions in France or abroad, or from public or private research centers.
L'archive ouverte pluridisciplinaire HAL, est destinée au dépôt et à la diffusion de documents scientifiques de niveau recherche, publiés ou non, émanant des établissements d'enseignement et de recherche français ou étrangers, des laboratoires publics ou privés. 


\title{
Synchronous Electric Charge and Induced Current Extraction (SECICE): a unified nonlinear technique combining piezoelectric and electromagnetic harvesting
}

\author{
G Lombardi ${ }^{1}$, M Lallart ${ }^{1}$ \\ ${ }^{1}$ Univ. Lyon, INSA Lyon, LGEF, EA682, F 6 9621, VILLEURBANNE, FRANCE \\ E-mail: mickael.lallart@insa-lyon.fr
}

October 2020

\begin{abstract}
In the framework of energy harvesting using as much as active parts as possible, this paper aims at proposing an electronic interface for hybrid (piezoelectric and electromagnetic) systems taking advantage of nonlinear treatments for significantly increasing the output current of an electromagnetic system while ensuring independence of the load for maximizing the final harvested power. The proposed technique, named Synchronous Electric Charge and Induced Current Extraction (SECICE) is derived from a combination of the Synchronous Electric Charge Extraction (SECE) and Synchronous Magnetic Flux Extraction (SMFE) schemes, respectively developed for piezoelectric and electromagnetic systems. The principle of the proposed technique lies in first transferring the piezoelectric charges to the electromagnetic system, and then extracting the electromagnetic energy through the SMFE approach. Compared to the classical SMFE scheme, the proposed scheme shows a significant increase in the output power, particularly for low-coupled/highly damped systems.
\end{abstract}

Keywords: Energy harvesting, piezoelectricity, electromagnetism, hybrid systems, nonlinear interface, synchronized switching

\section{Introduction}

In the era of the "Internet of Things" (IoT), billions of low-power consumption devices are going to be connected exponentially [8]. Such devices include sensors constituting an interconnected network used in different sectors such as healthcare, smart home, automotive, etc. The main issue and challenge come when these sensors are deployed in remote or hostile locations, beyond the reach of the electric grid. In such a situation, batteries are certainly a potential solution. However, due to their limited lifespan (due to ageing and self-discharge phenomena [2]) many logistical issues might arise when replacing them, particularly in sites where the task would be difficult, costly 
and sometimes even risky [6]. As an alternative, sustainable power generation may be achieved in harnessing energy that is already available in the environment and convert it into electrical energy, opening the doors to autonomous self-powered devices, subject of many studies for more than a decade [26], [30]. Among several feasible energy sources, like solar or thermal energy sources (to make few examples), ambient vibrations have the potential to meet the requirements for powering sensor nodes, as they are widely spread and characterized by high power density [35]. The process of converting vibrations into electrical energy is performed via several transduction mechanisms: both piezoelectricity $[27,29]$ and electromagnetism $[3,5]$ have been extensively studied for energy harvesting purposes as they both offer relatively high energy density and integration potentials. Vibrational energy harvesters still face several issues such as the frequency bandwidth and the energy conversion efficiency. While the issue of frequency bandwidth can be addressed with several implementations (both from a mechanical [7, 13, 14,31] and electrical $[4,20,25]$ point of view), this paper focuses on the energy conversion efficiency of vibrational energy harvesting systems, leaving aside the issue of frequency bandwidth.

With the goal of increasing the energy conversion efficiency of transduction mechanisms, nonlinear electronic elements, such as switches, can be added to the electrical circuit $[10,12,22]$. These so-called nonlinear electronic interfaces have been applied to both piezoelectric elements and electromagnetic ones, boosting the power gain of the transducers compared to the classical linear approaches (usually consisting of a linear load directly connected to the transducer or through a rectifier), especially for low-coupled electromechanical systems. With the intention of combining the power gain that can be obtained from such nonlinear interfaces and addressing the issue of the dependence between the load and the harvested power, the so-called Synchronous Electric Charge Extraction (SECE) has been proposed by Lefeuvre et al. [21]. This technique, applied to piezoelectric systems, has demonstrated to greatly enhance the electromechanical conversion efficiency while addressing the impedance matching issue. Playing with the magnetic flux/electrical charge duality [18], the SECE technique has been extended to electromagnetic microgenerators, namely the Synchronous Magnetic Flux Extraction (SMFE) [1].

In parallel, in order to further enhance the potential extraction of energy from ambient vibrations, researchers have started to integrate more than one conversion mechanism into the same structure, exploiting different or the same energy source using more than one transducer, making the system hybrid [16, 23, 24, 28, 32-34,37,38]. However, from an electrical point of view, there are several drawbacks when it comes to dealing with two transducers at a time, especially when considering electromagnetism and piezoelectricity:

- High difference in voltage/current outputs: additional DC/DC converters would be required in order to deal with the different magnitudes of current and/or voltage outputs;

- Peak powers of the two systems generally arising at different optimal load values: 
additional circuit stages dedicated to a common impedance matching would be required.

Both the two arguments introduce additional complexity to the circuit and, hence, extra power losses, explaining why the majority of the works in the literature have been mostly applied to the energy conversion process from a mechanical point of view (i.e. focusing on the mechanical optimization of the structure), rather than considering a dedicated electronic circuit (i.e. focusing on the energy extraction enhancement), due to the complexity at interfacing these systems electrically.

In order to smartly overcome the typical complications (from an electrical point of view) when considering hybrid electromagnetic-piezoelectric energy harvesters, a solution would be to replace passive electrical components with active ones on the same electrical interface [17]: for instance, a simple inductor can be replaced by an electromagnetic system while a capacitor can be replaced by a piezoelectric system. In this framework, this paper presents an electrical interface dedicated to the energy conversion enhancement and extraction of a hybrid energy harvesting system based on the coupling of electromagnetism and piezoelectricity, while proposing a nonlinear treatment of the electromagnetic output current. The main motivation behind the proposed hybrid approach (derived from a combination of the SECE and SMFE interfaces) lies in allowing a significant increase of the electromagnetic current by transferring the charge from the piezoelectric system before extracting the available electromagnetic energy. This strategy not only results in a final output power gain with respect to both the SMFE and the SECE techniques, but also allows to overcome the typical challenges (different voltage/current outputs and different load values that maximize the power) when it comes to interfacing electromagnetic and piezoelectric systems, thus proposing a valid solution for hybrid systems from an electrical point of view. Moreover, a relative independence from the load is also achieved (as long as the piezovoltage remains lower than half the final load voltage), as both the transducers are decoupled from the final harvesting stage.

The paper is organized as follows: Section 2 introduces the principles and the operations of the previously developed SMFE and SECE interfaces and the proposed hybrid one; Section 3 illustrates in detail the energy transfer analysis behind the proposed model, giving the output energy that can be extracted, firstly considering constant displacement magnitude and then taking into account the damping effect at resonance; a preliminary theoretical discussion is presented in Section 4; Section 5 aims at exposing the experiments carried out in order to validate such a model along with comparative discussion and analysis; finally, Section 6 briefly concludes the paper.

\section{Principles}

This section aims at presenting the basic principles behind the previously developed SMFE and SECE interfaces along with the introduction of the main principle and the 


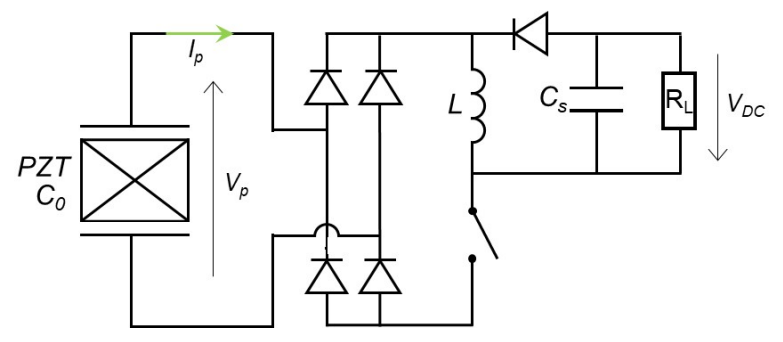

Figure 1: SECE interface $\left(C_{0}, I_{p}\right.$ and $V_{p}$ are respectively the piezoelectric clamped capacitance, current and voltage and $L$ is the coil inductance)

motivation of this work. The expression of the output harvested powers that can be achieved with each technique will be given in the next section.

\subsection{SECE}

The SECE nonlinear technique consists in switching the piezoelectric (PZT) element on an inductance at each extremum of the mechanical displacement. The circuit schematic is illustrated in figure 1. The piezoelectric system is initially open-circuited until the mechanical displacement reaches an extremum. At this point, the switch is closed and all the electrostatic energy stored on the clamped capacitance of the piezoelectric element is transferred to the coil. When all the electrical charges are transferred, the switch is opened again and the inductance discharges in the storing capacitor $C_{s}$ which is connected to a load $R_{L}$.

\section{2. $S M F E$}

Because of the magnetic flux/electrical charge duality that exists between piezoelectric and electromagnetic transducers, the previously exposed SECE technique has been extended to electromagnetic (EM) systems: in this case, the inductance (which in the SECE case is in series with a switch that is normally open) is replaced by a capacitance in parallel to a switch that is normally closed. Hence, the principle of the SMFE interface, shown in figure 2, consists in switching the electromagnetic element on a capacitor at each extremum of the current (which, without considering the coil losses, corresponds to the extremum of the mechanical displacement). Contrary to the SECE technique, the electromagnetic element is initially short-circuited until the mechanical displacement reaches an extremum. At this instant, the switch is opened and the electromagnetic energy is transferred to one of the storing capacitors (represented in the schematic by $C_{1}$ and $C_{2}$ ). The sudden opening of the switch leads to significant voltage levels, even when considering a small value of electromagnetic inductance, as they are still characterized by large values of current. The switch is again turned on at the completion of the energy transfer. 


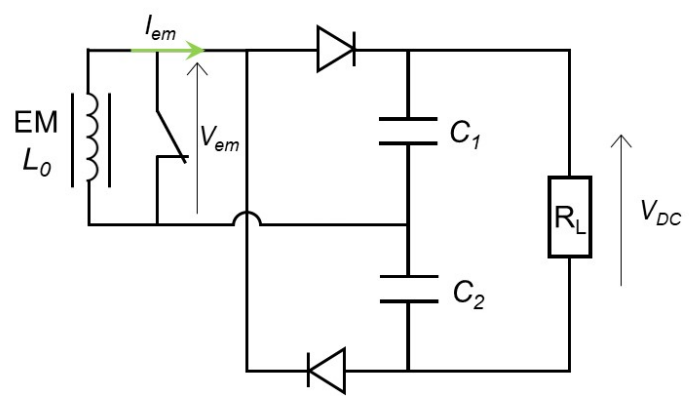

Figure 2: SMFE interface $\left(L_{0}, I_{e m}\right.$ and $V_{e m}$ are respectively the electromagnetic inductance, current and voltage)

\subsection{Hybrid model}

Seeing the similarities in the SMFE and SECE interfaces, the main motivation behind the proposed approach lies in allowing a significant current increase of the electromagnetic system by transferring the charge from the piezoelectric system before extracting the available electromagnetic energy, making active most of the components involved in the circuit. In order to do so, and starting from the SMFE interface, an additional switch $S_{1}$ is added between the piezoelectric and the electromagnetic system, as shown in figure 3a (where $S_{2}$ acts in the same way as the switch involved in the SMFE technique). When this switch $S_{1}$ is closed (and $S_{2}$ opened), the electrical energy stored on the piezoelectric capacitor $C_{0}$ is transferred into the inductor $L_{0}$ of the electromagnetic system (similarly to what happens in the SECE case), as per figure 3b. When the electric charge is completely transferred from the piezoelectric system, the switch $S_{1}$ is opened again (figure 3c) and the boosted electromagnetic current is transferred to the storing stage $\left(S_{2}\right.$ remains open in this phase), leading to the cancellation of the current (similarly to the SMFE case and in the second energy transfer step of the SECE). This current-boost is then responsible for the final enhanced extracted and harvested powers of the hybrid system. The strategy of transferring energy from one transducer to the other thus allows to smartly overcome the typical challenges when it comes to interfacing electromagnetic and piezoelectric systems. As a matter of fact, an electromagnetic system is characterized by high levels of current and low voltage, opposite to the piezoelectric element, distinguished by high levels of voltage and low current. Moreover, they are usually characterized by different values of load that maximizes the powers. Hence, extracting the available energy from the two systems separately (rather than focusing on the energy transfer and extraction as proposed in this work), would then lead to the employment of DC/DC converters and load adaptation stages, thus introducing additional complexity to the circuit and, consequently, additional power losses. 


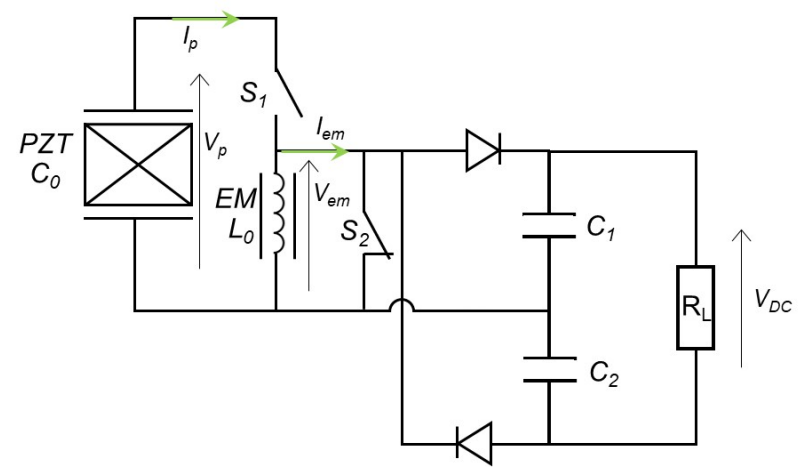

(a) Default configuration

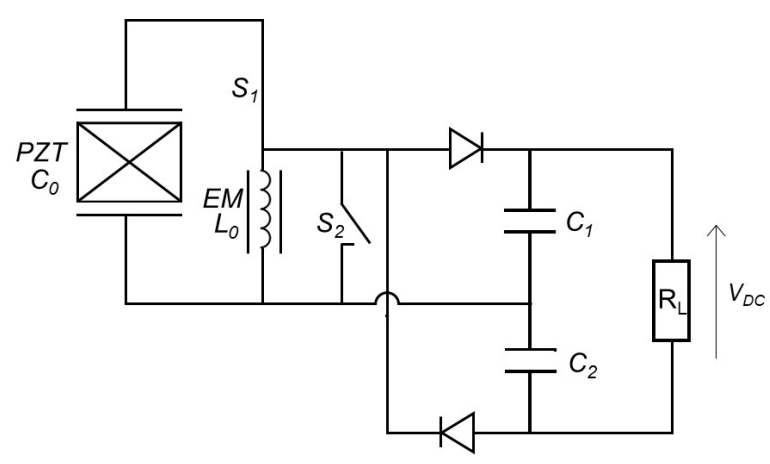

(b) Charge transfer configuration

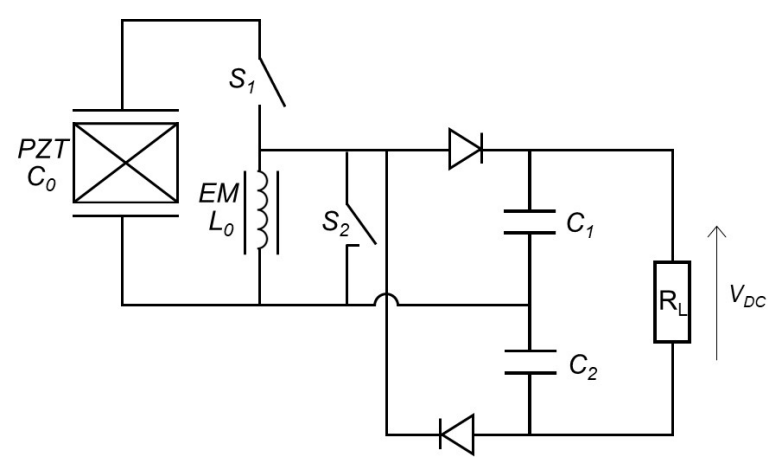

(c) Energy extraction configuration

Figure 3: Hybrid model circuit schematic (PZT and EM are respectively the piezoelectric and electromagnetic systems, $C_{0}$ and $L_{0}$ are respectively the piezoelectric clamped capacitance and electromagnetic inductance, $I_{p}$ and $I_{e m}$ are respectively the piezoelectric and electromagnetic currents, $V_{p}$ and $V_{e m}$ are respectively the piezoelectric and electromagnetic voltages) 


\section{Theoretical development}

Inertial microgenerators excited near one of their resonance frequencies are conventionally modelled as second-order mass-spring-damper systems [36]. Figure 4 shows the equivalent mechanical models for an electromagnetic generator (figure 4a) and for a piezoelectric generator (figure 4b), where $M$ represents the dynamic mass, $C$ the damper, modelling the mechanical losses, and $K^{E, H}$ is the spring, representing the stiffness of the system (when considering the piezoelectric element short-circuited while having the electromagnetic element open-circuited). Assuming that the system is excited by a force applied to the frame $F(t)=-M a(t)$, this excitation creates a relative displacement $u(t)$ between the mass and the support, which will allow the energy conversion thanks to the transducer (piezoelectric or electromagnetic). The first constitutive equation of (1) thus expresses the equation of motion of such an electromechanical system, resulting from the balance of the following forces: the inertial effect $(M \ddot{u})$, the viscous damping force $(C \dot{u})$, the kinetic inertial effect $\left(K^{E, H} u\right)$, the piezoelectric $\left(\alpha V_{p}\right.$, with $\alpha$ and $V_{p}$ being the piezoelectric force factor and output voltage, respectively) and the electromagnetic $\left(\beta I_{e m}\right.$, with $\beta$ and $I_{e m}$ being the electromagnetic force factor and output current, respectively) restoring forces.

Moreover, from an electrical point of view, an ideal electromagnetic generator can be represented as a voltage source (the source term being $\beta \dot{u}$ ) connected in series with an inductance $L_{0}$, while a piezoelectric generator behaves as a current source (the source term being $\alpha \dot{u}$ ) connected in parallel with a capacitance $C_{0}$ [18]. The proportionality between the produced electromagnetic current (resp. piezoelectric voltage) and the velocity of the mass $\dot{u}$ is represented by the force factor $\beta$ (resp. $\alpha$ ). Thus, the constitutive electromechanical equations describing the behaviour of the coupled electromagnetic-piezoelectric system are given by

$\ddagger$ the variables involved in the equation are as a function of time but for the sake of clarity the time dependency is not explicitly expressed unless otherwise stated.

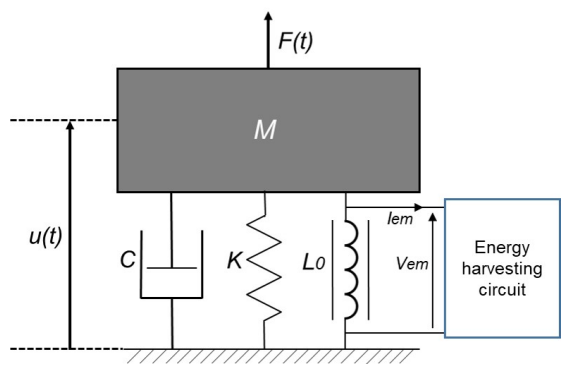

(a) Electromagnetic model

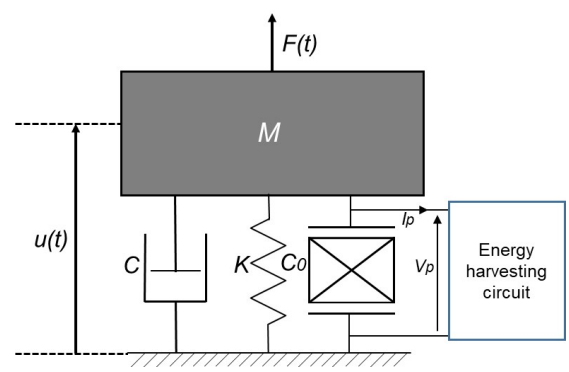

(b) Piezoelectric model

Figure 4: Equivalent mechanical models of piezoelectric and electromagnetic generators 


$$
\left\{\begin{array}{l}
M \ddot{u}+C \dot{u}+K^{E, H} u=F-\alpha V_{p}-\beta I_{e m} \\
I_{p}=\alpha \dot{u}-C_{0} \dot{V}_{p} \\
V_{e m}=\beta \dot{u}-L_{0} I_{e m}
\end{array}\right.
$$

where $u$ denotes the flexural displacement of the structure that is considered sinusoidal in the following, $V_{e m}$ and $V_{p}$ the electromagnetic and piezoelectric voltages respectively and $I_{e m}$ and $I_{p}$ the electromagnetic and piezoelectric currents respectively.

\subsection{Energy transfer analysis}

If the structure is supposed to be driven with a constant displacement magnitude (when no damping effect is taken into consideration), the equations describing the system are reduced to the electrical domain, yielding:

$$
\left\{\begin{array}{l}
I_{p}=\alpha \dot{u}-C_{0} \dot{V}_{p} . \\
V_{e m}=\beta \dot{u}-L_{0} \dot{I}_{e m}
\end{array}\right.
$$

Starting from this, and assuming that no connection occurs between the piezoelectric element and the harvesting stage (valid for loads that are large enough), the main phases characterizing the SECICE technique are the followings:

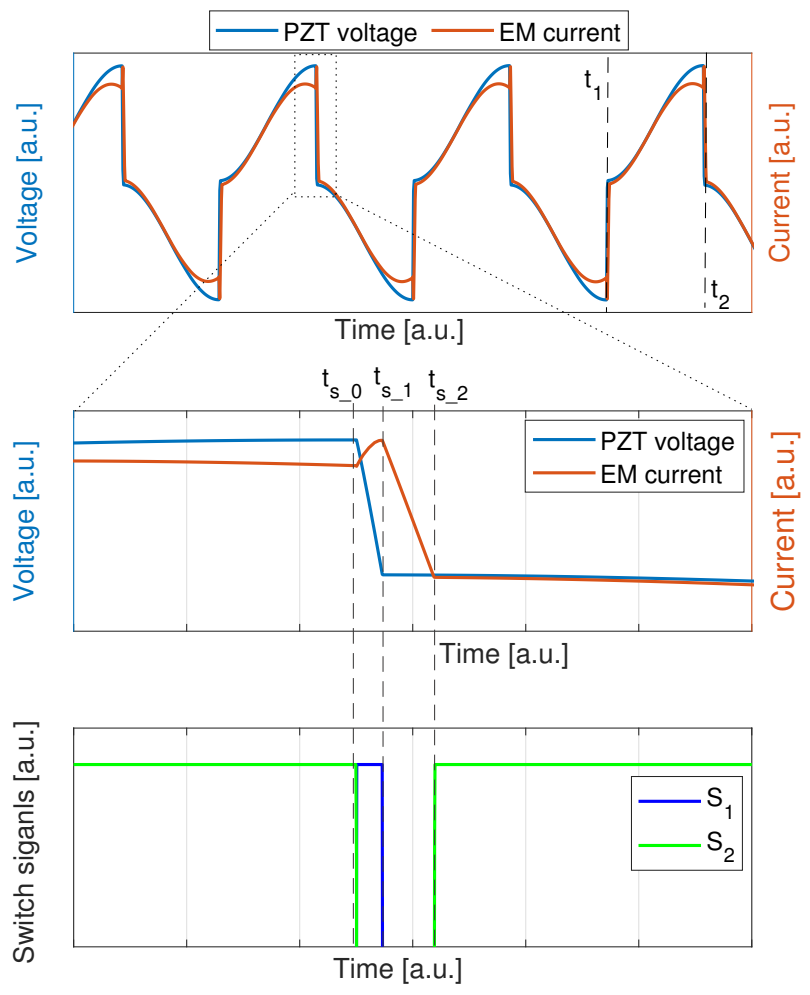

Figure 5: Voltage and current waveforms (a.u. stands for arbitrary unit) 
(i) Default configuration phase (figure 3a): the switches $S_{1}$ and $S_{2}$ are open and closed respectively; the electromagnetic system is short-circuited and the current varies proportionally to the mechanical displacement. On the other hand, the piezoelectric system is open-circuited and its voltage is related to the mechanical displacement:

$$
\left\{\begin{array}{l}
I_{p}=0 \Rightarrow \dot{V}_{p}=\frac{\alpha}{C_{0}} \dot{u} \\
V_{e m}=0 \Rightarrow I_{e m}=\frac{\beta}{L_{0}} \dot{u}
\end{array}\right.
$$

(ii) Charge transfer configuration phase (figure 3b): when the mechanical displacement reaches an extremum $\left(t=t_{s_{-} 0}\right)$, the switch $S_{1}$ is closed and $S_{2}$ is opened, as shown in figure $3 \mathrm{~b}$. The piezoelectric voltage $V_{M}$ and electromagnetic current $I_{M}$ corresponding to this instant can then be defined as:

$$
\left\{\begin{array}{l}
I_{M}=2 \frac{\beta}{L_{0}} u_{M} \\
V_{M}=2 \frac{\alpha}{C_{0}} u_{M}
\end{array}\right.
$$

where $u_{M}$ is the displacement magnitude. At this moment, the piezoelectric energy is transferred to the electromagnetic transducer, thus provoking the current peaks, as shown in figure 5. From an analytical point of view, the energy generation and transfer can be expressed as:

$$
\frac{1}{2} \eta_{p z t} C_{0} V_{M}^{2}+\frac{1}{2} L_{0} I_{M}^{2}=\frac{1}{2} L_{0} I_{1}^{2}
$$

where the first and the second term on the left side of the equation represents the energy available on the piezoelectric element and the energy available on the electromagnetic element, respectively. As the piezoelectric energy gets transferred to the inductance (with $\eta_{p z t}$ representing the losses within this energy transfer), the right term in equation (5) represents the final energy (related to the boosted electromagnetic current $I_{1}$ ) stored on the electromagnetic element at the end of the energy transfer.

(iii) Energy extraction configuration phase (energy transfer from the EM transducer to the harvesting stage, figure 3c): once all the piezoelectric energy has been transferred to the electromagnetic system (leading to the cancellation of the piezoelectric voltage), the switch $S_{1}$ is opened again and the piezoelectric element returns to open-circuit condition $\left(t=t_{s_{-}}\right)$. The switch $S_{2}$ continues to be opened so that the boosted electromagnetic current is transferred to one of the storing capacitances $\left(C_{1}\right.$ or $\left.C_{2}\right) . S_{2}$ will be closed again (at $t=t_{s_{-} 2}$ ) once the transfer of the electromagnetic energy is completed (leading to the cancellation of the electromagnetic current).

The total energy that can be thus extracted from the electromagnetic system can then be expressed as: 


$$
E_{\text {ext }}=\frac{1}{2} L_{0} I_{1}{ }^{2}=\frac{1}{2}\left(\eta_{p z t} \frac{4 \alpha^{2}}{C_{0}}+\frac{4 \beta^{2}}{L_{0}}\right) u_{M}{ }^{2}
$$

By multiplying equation (6) by twice the resonance frequency $f_{0}$ (as the harvesting events occur twice each vibration period), the extracted power can be obtained as:

$$
P_{e x t}=f_{0}\left(\eta_{p z t} \frac{4 \alpha^{2}}{C_{0}}+\frac{4 \beta^{2}}{L_{0}}\right) u_{M}{ }^{2}
$$

Finally, taking into account the losses that occur during the energy transfer from the electromagnetic system to the load (magnetic losses, diode losses, etc.), the expression of the harvested power yields:

$$
P_{\text {harv }}=f_{0}\left(\eta_{\text {sece }} \frac{4 \alpha^{2}}{C_{0}}+\eta_{\text {em }} \frac{4 \beta^{2}}{L_{0}}\right) u_{M}^{2}
$$

where $\eta_{e m}$ represents the efficiency of the energy transfer from the electromagnetic system to the storage stage and $\eta_{\text {sece }}$ represents the product between $\eta_{e m}$ and $\eta_{p z t}$, thus actually being equivalent to the efficiency of the energy transfer in the SECE technique. From this expression, one can note that the power does not depend on the load $R_{L}$ (as per the SMFE and SECE technique, exposed in the previous section) as long as the assumption regarding the non-direct connection between the piezoelectric system and the load is valid. This condition remains valid as long as the piezoelectric voltage magnitude is lower than half the load voltage $V_{D C}$ (as a full-wave voltage doubler circuit is employed), thus for:

$$
\begin{aligned}
V_{M} & \leq \frac{V_{D C}}{2} \\
\Rightarrow 2 \frac{\alpha}{C_{0}} u_{M} & \leq \frac{\sqrt{P_{\text {harv }} R_{C}}}{2}
\end{aligned}
$$

where $R_{C}$ is the critical value of load where the connection with the piezoelement starts to take place. Substituting the expression of the harvested power:

$$
\begin{gathered}
4 \frac{\alpha}{C_{0}} u_{M} \leq \sqrt{f_{0}\left(\eta_{\text {sece }} \frac{4 \alpha^{2}}{C_{0}}+\eta_{\text {em }} \frac{4 \beta^{2}}{L_{0}}\right) u_{M}^{2} R_{C}} \\
\Rightarrow 4 \frac{\alpha}{C_{0}} \leq f_{0}\left(\eta_{\text {sece }} \frac{\alpha^{2}}{C_{0}}+\eta_{e m} \frac{\beta^{2}}{L_{0}}\right) R_{C}
\end{gathered}
$$

The critical load value $R_{C}$ is thus reached when the following condition ends its validity:

$$
\frac{4}{C_{0} f_{0}\left(\eta_{\text {sece }}+\eta_{\text {em }} \frac{\beta^{2}}{L_{0}} \frac{C_{0}}{\alpha^{2}}\right)} \leq R_{C}
$$


Finally, it is possible to note that the power expression of equation (8) is equal to $P_{S E C E}+P_{S M F E}\left(P_{S E C E}\right.$ and $P_{S M F E}$ being defined as the harvested power expressions in the SECE and SMFE case):

$$
\begin{gathered}
P_{S M F E}=4 \eta_{e m} f_{0} \frac{\beta^{2}}{L_{0}}\left(\frac{F_{M}}{C \omega_{0}+\frac{4}{\pi} \frac{\beta^{2}}{L_{0}}}\right)^{2} \\
P_{S E C E}=4 \eta_{\text {sece }} f_{0} \frac{\alpha^{2}}{C_{0}}\left(\frac{F_{M}}{C \omega_{0}+\frac{4 \alpha^{2}}{\pi}}\right)^{2}
\end{gathered}
$$

\subsection{Constant excitation magnitude}

The energy extraction process from a coupled electromagnetic-piezoelectric harvesting system actually induces electrical damping effects which in turn leads to a reduction of the mechanical energy delivered to the structure. This yields to reducing the induced electromotive force and piezoelectric voltage and, hence, the total harvested power.

In order to derive the expression of the displacement as a function of the system parameters, multiplying the terms of the first constitutive equation of (1) by the velocity and integrating over half an oscillation period $\frac{T}{2}$ (i.e. between the time instants $t_{1}$ and $t_{2}$ of figure 5 ) yields (with the time variable made explicit):

$$
\begin{aligned}
\frac{T}{2} M\left[\dot{u}(t)^{2}\right]_{t_{1}}^{t_{2}}+C \int_{t_{1}}^{t_{2}} \dot{u}(t)^{2} \mathrm{~d} t+\frac{T}{2} K^{E, H}\left[u(t)^{2}\right]_{t_{1}}^{t_{2}}= \\
\qquad \int_{t 1}^{t 2} F(t) \dot{u}(t) \mathrm{d} u-\alpha \int_{t_{1}}^{t_{2}} V_{p}(t) \dot{u}(t) \mathrm{d} t-\beta \int_{t_{1}}^{t_{2}} I_{e m}(t) \dot{u}(t) \mathrm{d} t
\end{aligned}
$$

Considering the second equation of (2), the mechanical energy converted into electrical energy by the electromagnetic system is given by:

$$
\beta \int_{t_{1}}^{t_{2}} I_{e m}(t) \dot{u}(t) \mathrm{d} t=2 \frac{\beta^{2}}{L_{0}}\left(u_{M}\right)^{2}
$$

while the transferred energy of the piezoelectric element corresponds to the electrostatic energy available on the piezoelectric capacitance, defined as (referring to the first equation of (2)):

$$
\alpha \int_{t_{1}}^{t_{2}} V_{p}(t) \dot{u}(t) \mathrm{d} t=2 \frac{\alpha^{2}}{C_{0}}\left(u_{M}\right)^{2}
$$

At steady state and at the resonance, the variation of the potential elastic energy and the kinetic energy between the time instants $t_{1}$ and $t_{2}$ is null. Additionally, the force $\left(F_{M}\right)$ and the velocity are in phase, thus yielding: 


$$
\frac{\pi}{2} F_{M} u_{M}=\frac{\pi}{2} C u_{M}{ }^{2} \omega_{0}+2 \frac{\alpha^{2}}{C_{0}} u_{M}{ }^{2}+2 \frac{\beta^{2}}{L_{0}} u_{M}{ }^{2}
$$

and leading to the displacement amplitude, as per equation (20).

$$
u_{M}=\frac{F_{M}}{C \omega_{0}+\frac{4}{\pi}\left(\frac{\alpha^{2}}{C_{0}}+\frac{\beta^{2}}{L_{0}}\right)}
$$

By inserting the value of the displacement defined as equation (20) into equation (7) and equation (8), the extracted and harvested powers when considering the damping effect can then be expressed according to equation (21) and equation (22), respectively.

$$
\begin{gathered}
P_{\text {ext }} \text { damp } \\
=4 f_{0}\left(\eta_{p z t} \frac{\alpha^{2}}{C_{0}}+\frac{\beta^{2}}{L_{0}}\right)\left(\frac{F_{M}}{C \omega_{0}+\frac{4}{\pi}\left(\frac{\alpha^{2}}{C_{0}}+\frac{\beta^{2}}{L_{0}}\right)}\right)^{2} \\
P_{\text {harv }_{\text {damp }}}=4 f_{0}\left(\eta_{\text {sece }} \frac{\alpha^{2}}{C_{0}}+\eta_{\text {em }} \frac{\beta^{2}}{L_{0}}\right)\left(\frac{F_{M}}{C \omega_{0}+\frac{4}{\pi}\left(\frac{\alpha^{2}}{C_{0}}+\frac{\beta^{2}}{L_{0}}\right)}\right)^{2}
\end{gathered}
$$

It can be noted that equation (22) permits finding back the expressions of the SMFE and SECE powers by respectively considering $\frac{\alpha^{2}}{C_{0}}=0$ (i.e. null contribution of the piezoelectric system), following equation (23) and $\frac{\beta^{2}}{L_{0}}=0$ (i.e. null contribution of the electromagnetic system), following equation (24):

$$
\begin{aligned}
& P_{S M F E}=4 \eta_{e m} f_{0} \frac{\beta^{2}}{L_{0}}\left(\frac{F_{M}}{C \omega_{0}+\frac{4}{\pi} \frac{\beta^{2}}{L_{0}}}\right)^{2} \\
& P_{S E C E}=4 \eta_{\text {sece }} f_{0} \frac{\alpha^{2}}{C_{0}}\left(\frac{F_{M}}{C \omega_{0}+\frac{4 \alpha^{2}}{\pi}}\right)^{2}
\end{aligned}
$$

\section{Theoretical discussion}

Based on the previous model, the performance of the SECICE scheme (both considering constant displacement and constant excitation magnitudes) is analysed in this section. The results will be compared with the SMFE and the SECE interfaces, as well as the 
sole use of the electromagnetic or piezoelectric system directly connected to a load. To conduct the analysis, the following hypotheses are considered:

- the power losses and the electromagnetic current phase shift due to the coil resistance $r_{L}$ of the electromagnetic system are not taken into account;

- it will be considered valid the assumption of no direct connection between the piezoelectric system and the load during the charge transfer configuration;

- the efficiency values of the energy transfer $\eta_{p z t}$ and $\eta_{e m}$ are considered to be equal to 0.86 and 0.8 , respectively. These values have been chosen based on the typical energy transfer efficiency of previous works $[11,15]$ and the values obtained during the experimental validations.

\subsection{Constant displacement magnitude}

When considering the structure subjected to a constant displacement magnitude, figure 6a correlates the normalized harvested powers for the SECICE (following to equation (8)), SMFE (following to equation (14)) and pure resistive EM§ techniques as a function of the normalized resistance. The power for each case has been normalized w.r.t. the maximum power $\left(P_{E M}\right)_{\max }$ that can be obtained with the pure resistive EM approach, defined by:

$$
\left(P_{E M}\right)_{\max }=\frac{1}{4} \frac{\beta^{2} \omega_{0}}{L_{0}} u_{M}^{2}
$$

while the resistance has been normalized w.r.t. the optimal load $\left(R_{E M}\right)_{\text {opt }}$ that maximises the power in the pure resistive EM approach:

$$
\left(R_{E M}\right)_{o p t}=L_{0} \omega_{0}
$$

Moreover, defining the ratio between the piezoelectric and the EM energy conversion capabilities, as:

$$
\text { Ratio }=\frac{\alpha^{2}}{C_{0}} / \frac{\beta^{2}}{L_{0}}
$$

in this particular example, the value of ratio has been fixed to 1.4. This value corresponds to the value obtained with the experimental apparatus that will be presented in section 5. The figure 6a clearly shows how the SECICE technique allows achieving greater power outputs both with respect to the SMFE and pure resistive EM techniques. In particular, with the proposed hybrid technique, and the value of ratio chosen for this particular example, 5.4 times more power can be harvested compared to the case of pure resistive EM, while achieving a gain of 1.7 w.r.t. the SMFE technique.

Similarly, figure $6 \mathrm{~b}$ depicts the normalized harvested powers for the SECICE (following to equation (8)), SECE (following to equation (15)) and pure resistive PZT\|

$\S$ i.e. when directly connecting the electromagnetic system to a resistor

\| i.e. when connecting the piezoelectric system directly to a resistor 

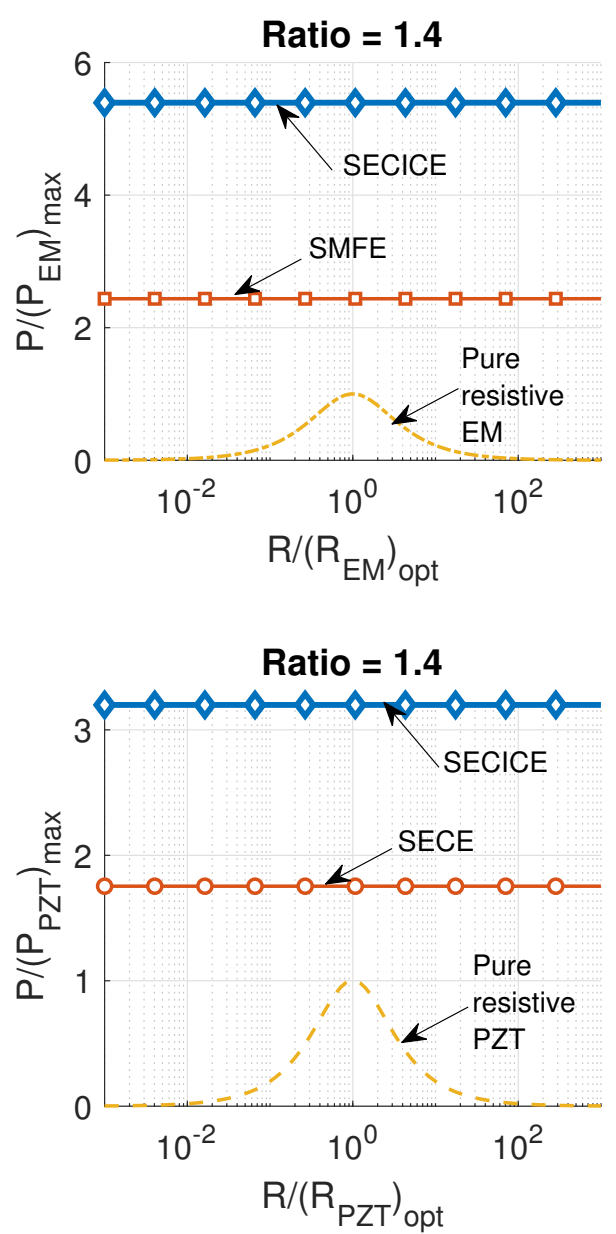

Figure 6: a) Normalized harvested powers (w.r.t. (a) the maximal harvested power in the pure resistive EM approach $\left(P_{E M}\right)_{\max }$ and (b) the maximal harvested power in the pure resistive PZT approach $\left.\left(P_{P Z T}\right)_{\max }\right)$ as a function of the normalized load (w.r.t. (a) the optimal load in the Pure resistive EM approach $\left(R_{E M}\right)_{o p t}$ and (b) the optimal load in the pure resistive PZT approach $\left.\left(R_{P Z T}\right)_{\text {opt }}\right)$ for different techniques

techniques as a function of the normalized resistance. For this plot, the power for each case has been normalized w.r.t. the maximum power $\left(P_{P Z T}\right)_{\max }$ that can be obtained with the pure resistive PZT approach defined by:

$$
\left(P_{P Z T}\right)_{\max }=\frac{1}{2} \frac{\alpha^{2} \omega_{0}}{C_{0}} u_{M}^{2}
$$

while the resistance has been normalized w.r.t. the optimal load $\left(R_{P Z T}\right)_{\text {opt }}$ that maximises the power in the pure resistive PZT approach:

$$
\left(R_{P Z T}\right)_{o p t}=\frac{1}{C_{0} \omega_{0}}
$$

The same value of ratio (1.4) was used for this plot as well. In this case, the SECICE technique allows achieving 3.2 more power than in the case of pure resistive 
PZT technique and a gain of 1.75 is obtained w.r.t. SECE technique. In addition, both figure $6 \mathrm{a}$ and figure $6 \mathrm{~b}$ demonstrate that the load does not need to be tuned to maximize the power, assuming, as previously stated, non-direct connection between the pizeoelement and the load during the charge transfer configuration, which may not be fulfilled for low load values $\uparrow$.

In order to better understand the power gain that can be achieved with the proposed hybrid technique w.r.t. the SMFE one, figure 7a depicts the extracted power normalized w.r.t. the maximum extracted power in the SMFE interface as a function of the ratio (i.e. as a function of the increase of the electromechanical capabilities of the involved transducers), as defined in equation (27). From the figure, the relation between the power gain w.r.t. the SMFE technique and the ratio visibly shows that increasing the electromechanical capabilities of the transducers (and more particularly, of the piezoelectric element) leads to higher power gains when employing the proposed SECICE scheme. This is due to the augmentation of piezoelectric charges that are transferred to the electromagnetic system.

With the intention of highlighting the interests of the SECICE technique in relation to the SECE one, the power gains in figure $7 \mathrm{~b}$ have been plotted as a function of the inverse of the ratio, defined as:

$$
\text { Ratio }^{-1}=\frac{\beta^{2}}{L_{0}} / \frac{\alpha^{2}}{C_{0}}
$$

Similarly, to figure 7a, in figure $7 \mathrm{~b}$ the trend of the power gain w.r.t. the SECE technique increases dramatically with the increase of ratio $^{-1}$. In this case, higher power gains are reached when increasing the electromechanical capabilities of the electromagnetic system. This is because, contrarily to the SECE interface, the inductor is actively participating in the energy harvesting process, boosting the final current of the global system.

From both the plots in figure 7, the power achieved with the proposed hybrid technique is higher than the maximum powers that can be extracted from either the SECE or SMFE schemes, demonstrating the benefit, in terms of power outputs and performance of the system, of replacing typical electronic passive components (e.g. capacitors/inductors) with active ones (e.g. piezoelements/electromagnetic transducers).

\subsection{Constant excitation magnitude}

When subjecting the structure to a constant excitation magnitude (allowing to take into account the damping effect), figure 8a shows the evolution of the powers (following to equations (21) and (22) for the SECICE technique, equation (23) for the SMFE technique and equation (24) for the SECE technique), normalized with respect to the

I in most of the cases, for self-powered systems it is unlikely to have low values of input impedance. 

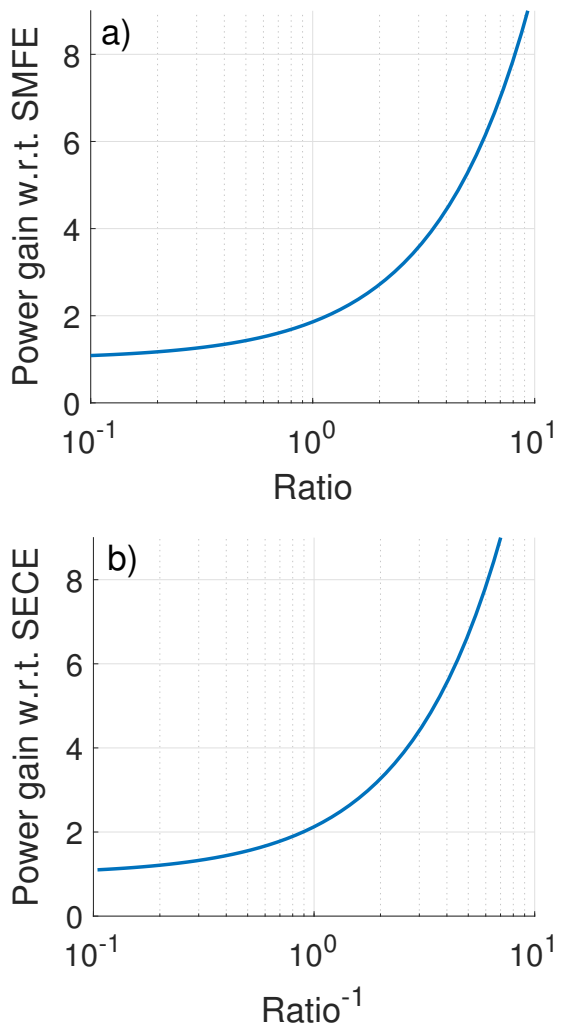

Figure 7: Harvested power gains w.r.t. the SMFE and SECE techniques as a function of (a) the ratio between the piezoelectric and the electromagnetic energy conversions and (b) the inverse of the ratio

power limit that can be achieved when considering electromechanical systems (being subjected to internal losses), defined as [9]:

$$
P_{l i m}=\frac{F_{m}^{2}}{8 C}
$$

The normalized power is plotted as a function of the product of the squared coupling piezoelectric coefficient $k_{p}{ }^{2}$ (denoting the available energy that can actually be converted) and the mechanical quality factor of the electromechanical structure $Q_{m}$ (representing the available amount of energy entering in the piezoelectric structure). The power trends of the hybrid SECICE technique can be explained as following: for low values of $k_{p}{ }^{2} Q_{m}$, the contribution of the piezoelectric element is negligible and the technique is equivalent to the SMFE interface. As the piezoelectric coupling level increases, the hybrid technique outperforms the single-transducer harvesting systems, exhibiting an optimal range of coupling values for which the proposed studied technique appears to be of particular interest ("optimal range" area in the graph). This area corresponds to coupling values ranging from $k_{p}{ }^{2} Q_{m}=0.01$ to $k_{p}{ }^{2} Q_{m}=0.28$, consistent with common values for vibrational energy harvesters [1]. After a certain limit of the FoM $k_{p}{ }^{2} Q_{m}$, the damping effect due to the combination of the two energy harvesting 


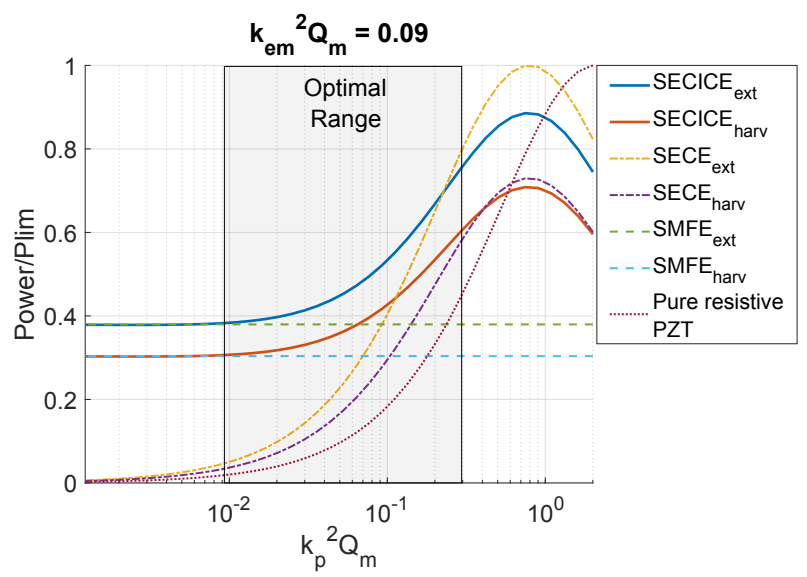

(a) Normalized maximum harvested power (w.r.t. the power limit $P_{\text {lim }}$ ) for different techniques versus the figure of merit $k_{p}{ }^{2} Q_{m}$ when taking into account the damping effect

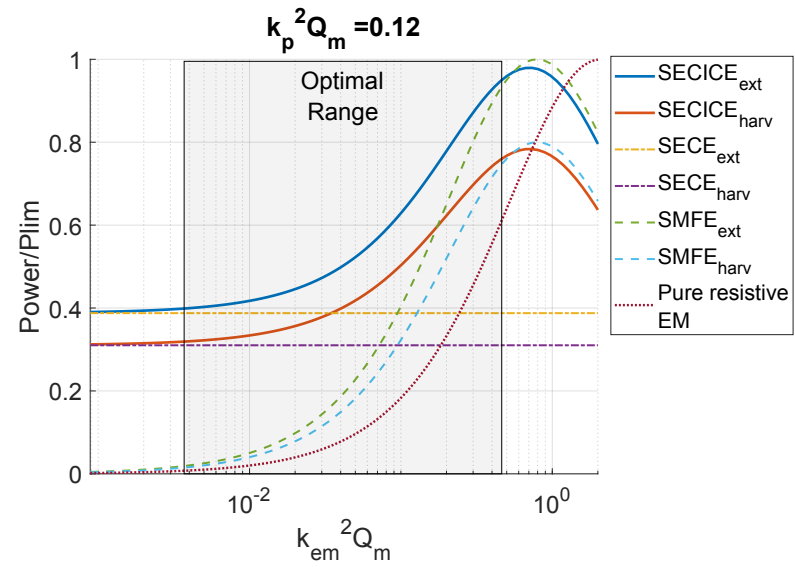

(b) Normalized maximum harvested power (w.r.t. the power limit $P_{\text {lim }}$ ) for different techniques versus the figure of merit $k_{e m}{ }^{2} Q_{m}$ when taking into account the damping effect

Figure 8: Normalized maximum harvested power (w.r.t. the power limit) for different techniques

transducers becomes more relevant, impacting the dynamics of the electromagnetic transducer and thus limiting the harvested power.

The power trend of the SECICE technique is confirmed by figure 8b, plotting the power as a function of the figure of merit ${k_{e m}}^{2} Q_{m}$, where ${k_{e m}}^{2}$ represents the squared coupling coefficient of the electromagnetic element. The optimal range for which the proposed technique appears to be of particular interest has been highlighted in this figure as well, from $k_{e m}{ }^{2} Q_{m}=0.005$ and $k_{e m}{ }^{2} Q_{m}=0.45$, compatible with coupling values when considering small scale electromagnetic energy harvesting systems [1].

Focusing on the behaviour of the power output with the proposed SECICE technique with respect to the SECE one, figure 9a depicts the evolution of the normalized 
power (normalized w.r.t. the power limit, defined in equation (31)) versus the FoM $k_{p}{ }^{2} Q_{m}$ for different values of $k_{e m}{ }^{2} Q_{m}$. In order to analyse the power gain with respect to the SMFE technique, the equivalent power output (obtained for the same value of $k_{e m}{ }^{2} Q_{m}$ ) that can be obtained without the contribution of the piezoelectric system is also plotted. Moreover, the optimal range area in which the proposed technique appears to be particularly beneficial is indicated in each plot.
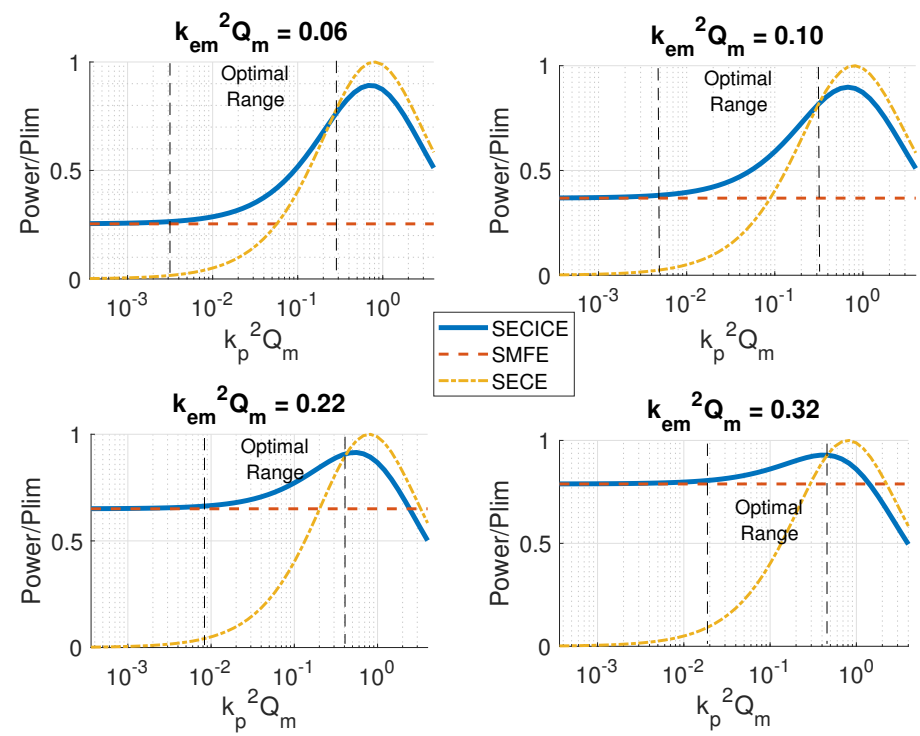

(a) Normalized maximum harvested power (w.r.t. the power limit $\left.P_{\text {lim }}\right)$ as a function of the figure of merit ${k_{p}}^{2} Q_{m}$ for different values of $k_{e m}^{2} Q_{m}$
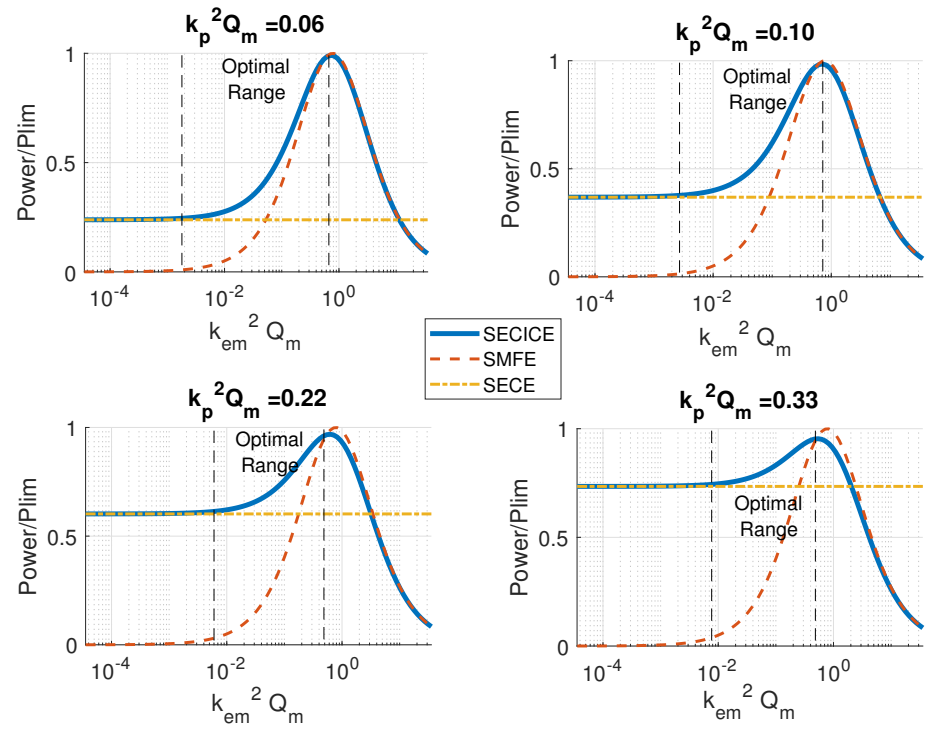

(b) Normalized maximum harvested power (w.r.t. the power limit $\left.P_{\text {lim }}\right)$ as a function of the figure of merit $k_{e m}{ }^{2} Q_{m}$ for different values of $k_{p}{ }^{2} Q_{m}$

Figure 9: Normalized maximum harvested power (w.r.t. the power limit) for different techniques 


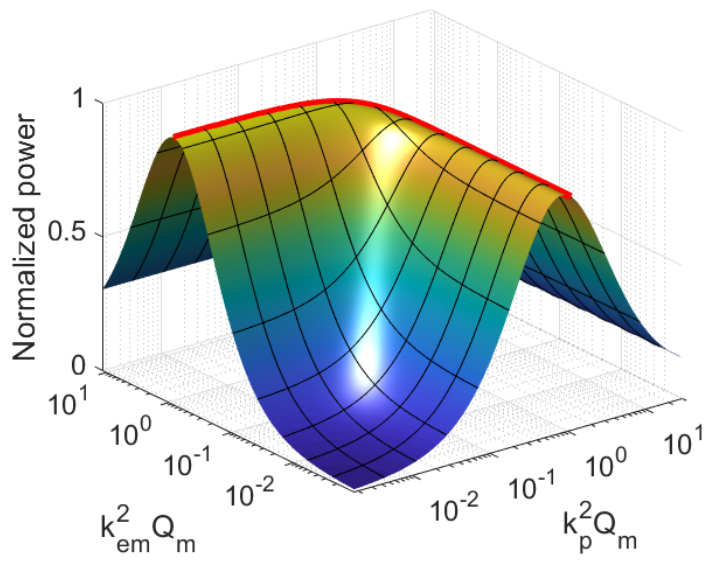

Figure 10: Normalized maximum harvested power (w.r.t. the power limit $P_{\text {lim }}$ ) as a function of the figures of merit $k_{e m}{ }^{2} Q_{m}$ and $k_{p}{ }^{2} Q_{m}$

The figure clarifies how higher power gains (particularly w.r.t. the power that can be reached with the SMFE technique) can be achieved for low coupled/highly damped structures, noting that the optimal range area becomes wider as the coupling of the electromagnetic transducer decreases. On the other hand, as the coupling of the system increases, the damping effect associated with the losses during the piezoelectric to the EM energy transfer step in the SECICE limits the output power of the proposed interface, leading to higher power outputs in favour of the SECE and SMFE techniques (as the damping effect in these techniques is limited to the use of one active system only - piezo or EM - instead of two).

The same behaviour can be observed in figure $9 \mathrm{~b}$, comparing the power output of the proposed hybrid technique with the equivalent power output (obtained for the same $k_{p}{ }^{2} Q_{m}$ values) that can be obtained with the SECE and SMFE techniques. Once again, higher power gains can be observed when the coupling of both the electromechanical systems is low, particularly exhibiting wider optimal ranges of power gain for low values of piezoelectric coupling.

In addition, figure 9 demonstrates that in hybrid systems it is important to choose the right trade-off between the electromechanical capabilities of the active systems and the amount of power to be harvested. For this particular electrical configuration, the right compromise falls within the electromechanical coupling range typical of small-scale energy harvesting systems.

Finally, figure 10 depicts the evolution of the normalized output power (with respect to the power limit) as a function of the figures of merit $k_{e m}{ }^{2} Q_{m}$ and $k_{p}{ }^{2} Q_{m}$, confirming the statements established so far: for each electromechanical coupling exists an optimum value (highlighted in red) of power that can be reached, while for high values of coupling (either piezoelectric or electromagnetic), the power decreases similarly to SECE and SMFE approaches. 


\section{Experimental validations}

In this section, the theoretical evaluations of the proposed technique discussed so far are here proposed to be experimentally demonstrated. The obtained results are compared with the SMFE technique. An equivalent evaluation of the performance of SECE technique (being a well-established technique) is also theoretically derived and discussed comparatively based on the experimental parameters defining the structure.

\subsection{Experimental set-up}

The experimental set-up used in this work is illustrated in figure 11a. The electromagnetic generator is composed of a coil with a U-shaped ferrite core (the coil is wrapped around one of the legs of the ferrite). The solution of using a ferrite core allows achieving a higher inductance compared to generators composed of a simple air coil, thus yielding a value of reactance $L_{0} \omega_{0}$ that permits to neglect the coil resistance in the present experiments. Additionally, the coil is enclosed inside a $0.25 \mathrm{~mm}$-thick nickeliron soft ferromagnetic alloy which acts as a shield against the magnetic fields from the surroundings thanks to its high permeability. This also allows the magnetic flux lines to be canalised within the magnetic circuit of the electromagnetic system. Both the ferrite core and the additional magnetic shield contribute to obtaining higher values of reactance within the electromagnetic generator, explaining why, at the operating frequency, the coil losses have not been taken into account in the model. The coil along with its ferrite core is placed in front of a piezoelectric buzzer, as shown in figure 11b. In order to allow the coupling of the two microgenerators, a permanent magnet (NdFeB) is placed on the surface of the piezoelectric buzzer. As the magnet moves along the axis of the coil, the output electrical signals of the generators are at the same frequency. It has to be noted that the magnetic orientation of the NdFeB magnet, as shown in figure $11 \mathrm{~b}$, has been chosen so that the piezoelectric system is not excessively clamped by the magnet itself (which would otherwise occur when mounting the magnet with one of the magnetic poles on the piezoelectric surface). Despite this, a notable flux variation is still seen by the coil thanks to the magnetic circuit of the ferrite core.

Finally, the hybrid system composed of the piezoelectric buzzer and the electromagnetic generator is placed on a shaker providing a base sinusoidal excitation. The sinusoidal signal is generated by a RTI 1104 dSpace card (which is also used to trigger the switches commands at the extrema of the mechanical displacement) and a voltage amplifier. The connections between the dSpace card, the switches (composed of two NMOS transistors (2N7000) controlled through an optocoupler) and the transducers is illustrated in figure 11c. Hence, in this case the switches used in the SECICE interface were externally powered and controlled (as the purpose of this paper is to propose the working principle of the hybrid SECICE circuit), although a fully selfpowered switching interface could be investigated in future works, adapting what has been recently developed for piezoelectric systems [25]. The waveforms of the output currents and voltages are displayed in an oscilloscope while a laser displacement sensor 


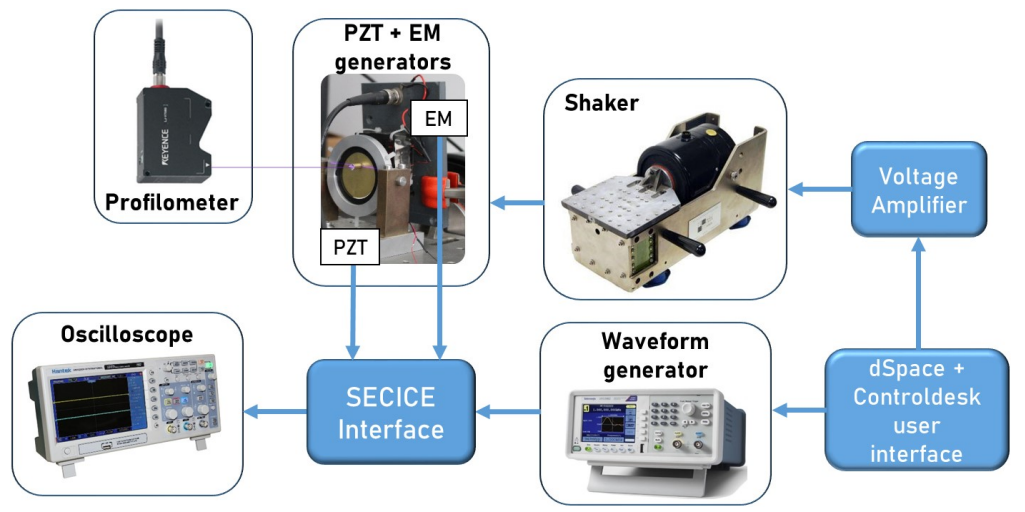

(a) Experimental set-up schematic

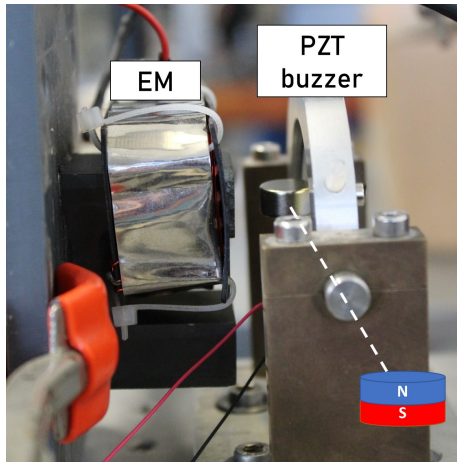

(b) EM and PZT transducers

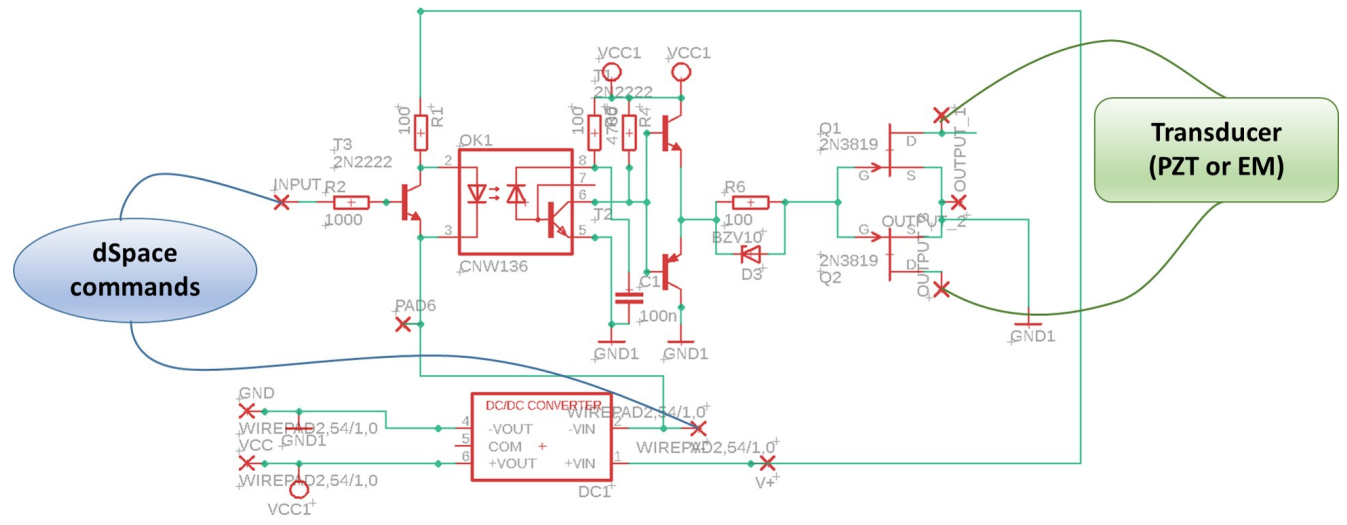

(c) Switching circuit and connections

Figure 11: Experimental set-up

(Keyence LJ-V7000) monitors the mechanical displacement. The $D C$ voltage seen on the load $R_{L}$ is measured using a digital multimeter.

\subsection{Model parameters identification}

In order to estimate the main parameters characterising the hybrid structure, preliminary experimental identifications have been conducted yielding the values listed in table 1. The electromagnetic force factor $\beta$, defined as $L_{0} \times\left(I_{S C} / u_{M}\right)$, has been 
obtained by measuring the short-circuit current $I_{S C}$, the displacement magnitude $u_{M}$ and the coil inductance $L_{0}$. Similarly, measuring the open circuit output voltage $V_{O C}$, the displacement magnitude $u_{M}$ and piezoelectric clamped capacitance $C_{0}$, the piezoelectric force factor $\alpha$ can be determined as $C_{0} \times\left(V_{O C} / u_{M}\right)$. The coil inductance $L_{0}$ and resistance $r_{L}$ and the piezoelectric capacitance $C_{0}$ have been measured with a LCR meter. The short-circuit and the open-circuit electromagnetic resonance frequencies (when short-circuiting the piezoelectric system) allow deriving the electromagnetic coupling $k_{e m}{ }^{2}$, obtaining an experimental value of $k_{e m}{ }^{2}=0.0057$. Such a low value of electromechanical coupling is actually common for energy harvesting applications in small-scale devices [1]. Conversely, the piezoelectric coupling coefficient $k_{p}{ }^{2}$ is derived from the resonance frequencies when the piezoelectric element is either short-circuited or left open-circuited while leaving the electromagnetic transducer open-circuited, giving an experimental value of ${k_{p}}^{2}=0.05$, which is, once again, compatible with the application of

Table 1: Model parameters (PZT and EM respectively refer to piezoelectric and electromagnetic transducers; "oc" and "sc" respectively stand for "open-circuit" and "short-circuit")

\begin{tabular}{lr}
\hline Parameter & Value \\
\hline PZT clamped capacitance, $C_{0}$ & $67.85 \mathrm{nF}$ \\
EM coil inductance, $L_{0}$ & $27.55 \mathrm{mH}$ \\
EM coil resistance, $r_{L}$ & $5.8 \Omega$ \\
PZT force factor, $\alpha$ & $0.0052 \mathrm{NV}^{-1}$ \\
EM force factor, $\beta$ & $1.25 \mathrm{~N}^{-1}$ \\
Mechanical quality factor, $Q_{m}$ & 42 \\
Resonance frequency, PZT sc, EM oc $f_{E, H}$ & $162.15 \mathrm{~Hz}$ \\
Resonance frequency, PZT oc, EM oc $f_{D, H}$ & $165.33 \mathrm{~Hz}$ \\
Resonance frequency, PZT sc EM sc $f_{E, B}$ & $162.63 \mathrm{~Hz}$ \\
PZT coupling coefficient, $k_{p}{ }^{2}$ & 0.0502 \\
EM cupling coefficient, $k_{e m}{ }^{2}$ & 0.0057 \\
Stiffness (PZT sc, EM oc), $K^{E, H}$ & $1.849 \times 10^{4} \mathrm{Nm}^{-1}$ \\
Stiffness (PZT oc, EM oc), $K^{D, H}$ & $1.847 \times 10^{4} \mathrm{Nm}^{-1}$ \\
Stiffness (PZT sc, EM sc), $K^{E, B}$ & $1.849 \times 10^{4} \mathrm{Nm}^{-1}$ \\
Stiffness (PZT oc, EM sc), $K^{D, B}$ & $1.849 \times 10^{4} \mathrm{Nm}^{-1}$ \\
Dynamic mass, $M$ & $15 \mathrm{~g}$ \\
Damping coefficient, $C$ & $0.393 \mathrm{Nm}^{-1} s^{-1}$ \\
\hline
\end{tabular}


Table 2: Boundary electrical conditions

\begin{tabular}{lcc}
\hline Condition & Piezoelectric transducer & Electromagnetic transducer \\
\hline$D, H$ & open-circuited & open-circuited \\
$E, H$ & short-circuited & open-circuited \\
$E, B$ & short-circuited & short-circuited \\
$D, B$ & open-circuited & short-circuited \\
\hline
\end{tabular}

nonlinear interfaces for small-scale energy harvesting systems [19]. The fourth stiffnesses are obtained by means of the following relations:

$$
\begin{aligned}
& K^{D, H}= \frac{\alpha^{2}}{C_{0}} \frac{f_{D, H}{ }^{2}}{f_{D, H}{ }^{2}-f_{E, H}{ }^{2}}=\frac{\alpha^{2}}{C_{0}} \frac{1}{{k_{p}}^{2}} \\
& K^{E, H}=K^{D, H}-\frac{\alpha^{2}}{C_{0}} \\
& K^{E, B}=\frac{\beta^{2}}{L_{0}} \frac{f_{E, H}{ }^{2}}{f_{E, B}{ }^{2}-f_{E, H}{ }^{2}}=\frac{\beta^{2}}{L_{0}} \frac{1}{k_{e m}{ }^{2}} \\
& K^{D, B}=K^{E, B}-\frac{\beta^{2}}{L_{0}}
\end{aligned}
$$

where the meaning of subscripts $D, H, E, H, E, B$ and $D, B$ is summarized in table 2. Finally, the remaining mechanical parameters (dynamic mass and the damping coefficient) are obtained as:

$$
\begin{gathered}
M=\frac{K^{E, H}}{\omega_{E, H^{2}}} \\
C=\frac{M \omega_{D, H}}{Q_{m}}
\end{gathered}
$$

where $Q_{m}$ is the mechanical quality factor with respect to the $-3 \mathrm{~dB}$ bandwidth.

\subsection{Results and discussion}

When considering constant displacement magnitude, the structure is driven at its resonance frequency with an imposed displacement magnitude of $u_{M}=95 \mu \mathrm{m}$. Figure 12 illustrates the experimental waveforms for the SMFE and the proposed SECICE technique when considering a load value of $500 \mathrm{k} \Omega$. The blue lines represent the current waveforms measured on the electromagnetic system, the red line represents the rectified voltage (measured on the final load $R_{L}$ ) while the green line represents the piezoelectric voltage waveform (present only in the hybrid interface). The figure clearly shows the generation of the electromagnetic current peaks in the hybrid technique thanks to the 

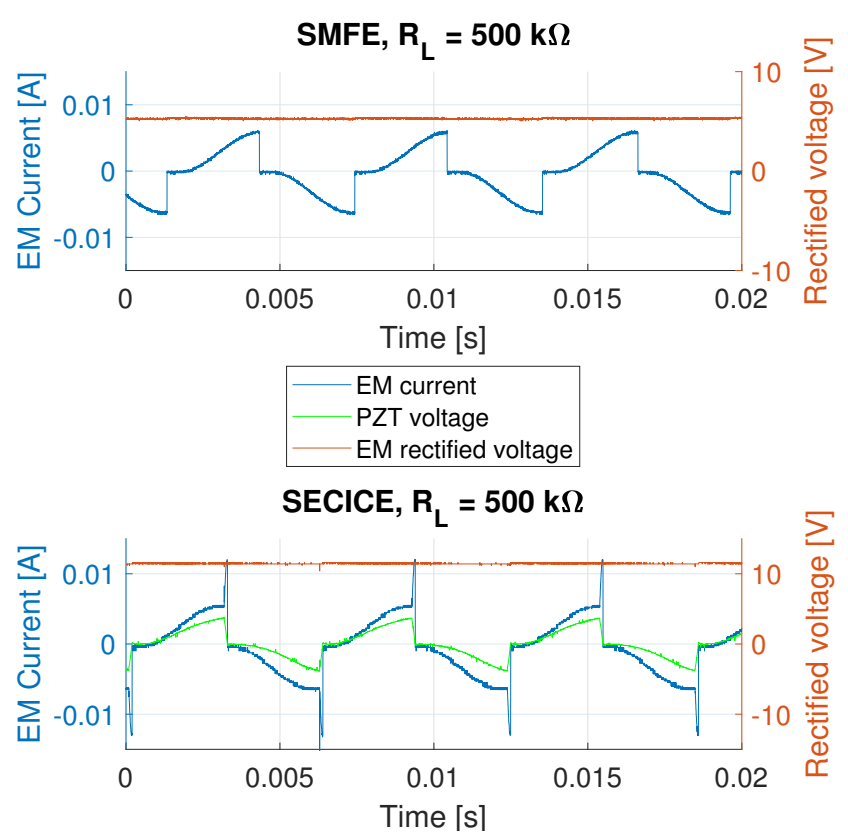

Figure 12: Experimental waveforms

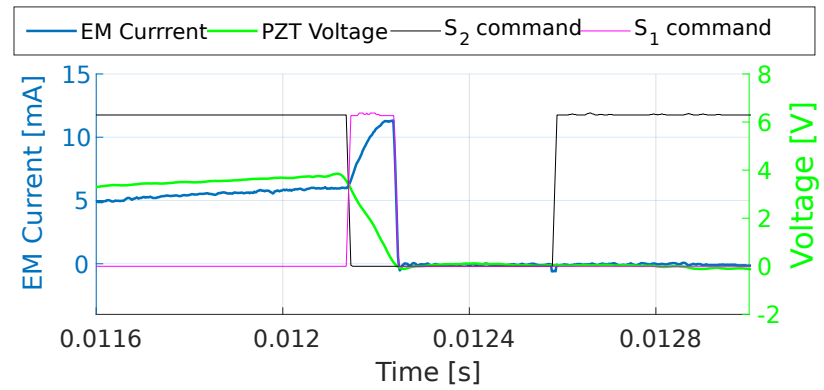

Figure 13: Enlargement of experimental waveforms

charge transfer from the piezoelectric system. The boosted current (equivalent to twice the current values of the SMFE technique) is then responsible for the enhancement of the rectified DC voltage and, consequently, of the final power.

Figure 13 depicts the enlargement of the waveforms along with the switching signals $S_{1}$ (purple line) and $S_{2}$ (black line). This figure experimentally demonstrates the working principle of the proposed hybrid interface: when the switch $S_{1}$ is opened and $S_{2}$ is closed, the absolute values of the EM current (blue line) and piezoelectric voltage (green line) are both increasing (default configuration - figure 3a). When an extremum of the displacement is reached, $S_{1}$ is closed and $S_{2}$ is opened so that the piezoelectric charges are transferred to the EM system (charge transfer configuration - figure $3 \mathrm{~b}$ ), leading to the cancellation of the piezoelectric voltage and a boost of the EM current, as shown in the figure. Once all of the piezoelectric charges have been transferred, $S_{1}$ is opened again while $S_{2}$ continues to be opened in order to allow the transfer of the boosted EM current to one of the storing capacitors (energy extraction configuration - figure $3 \mathrm{a}$ ). 

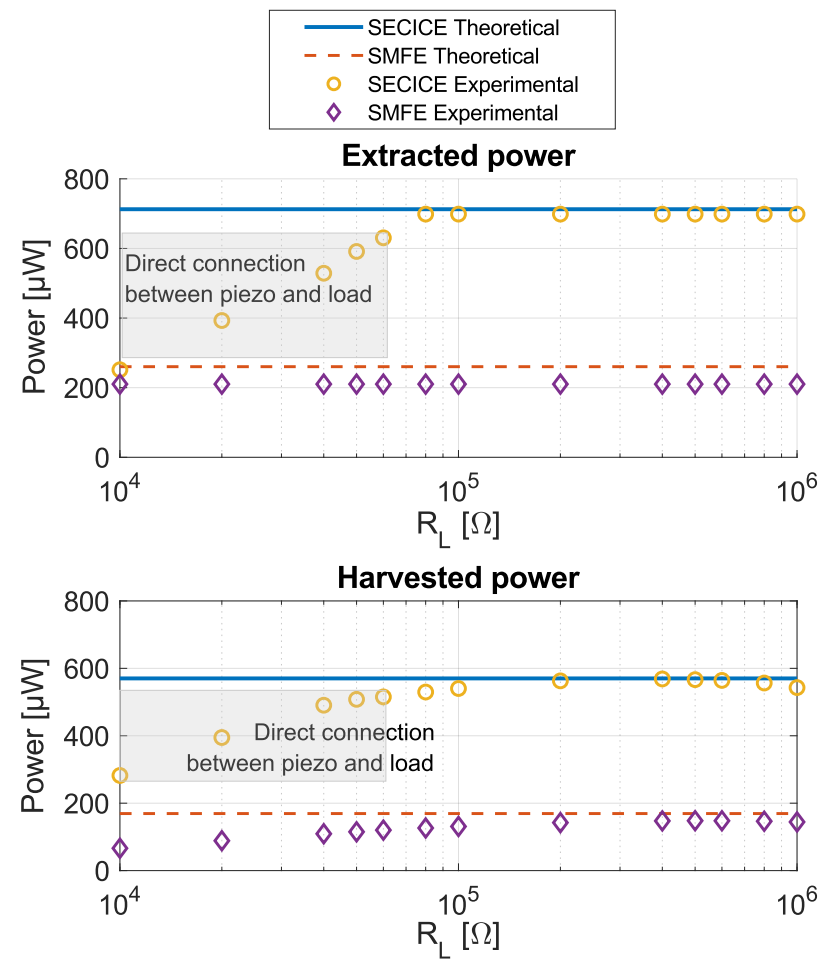

Figure 14: Experimental and theoretical harvested and extracted powers in the constant displacement magnitude case when considering an additional capacitor of $0.22 \mu F$ connected in parallel to the piezoelectric system (the grey zone represents the case of direct connection between the piezoelectric element and the storage stage - due to the low values of load - during the charge transfer configuration)

The experimental results concerning the extracted and harvested powers for the two techniques are plotted in figure 14 (when considering an additional capacitor of $0.22 \mu \mathrm{F}$ connected in parallel to the piezoelectric system), demonstrating a good agreement with the theoretical predictions. The experimental extracted power has been measured by recording the peak current values $I_{\text {peak }}$ with the current probe and proceeding with the following relation:

$$
P_{\text {ext }}=f_{0} L_{0} I_{\text {peak }}^{2}
$$

while for the calculation of the experimental harvested power, the following relation has been used:

$$
P_{h a r v}=\frac{V_{D C}{ }^{2}}{R_{L}}
$$

where $R_{L}$ is the final resistive load, which is made variable (from $100 \Omega$ to $10 \mathrm{M} \Omega$ ), and $V_{D C}$ is the rectified voltage measured across the load. As expected, the SECICE technique allows achieving higher power outputs, with a power gain of 4 compared with the maximal harvested power in the SMFE case. For the latter, the efficiency of the 
energy transfer from the electromagnetic system to the final load $\eta_{e m}$ was evaluated to be 0.68 , thus less than the efficiency of the hybrid technique (0.8), probably due to the lower values of current involved. Moreover, it can be noted from figure 14 that the harvested and extracted powers are roughly independent of the resistor value, with the exception of relatively low resistance values ("direct connection between piezo and load" area highlighted in grey), for which the assumption that the piezoelement is not conducting to the storage stage is not fulfilled (hence, there is a direct connection between the piezoelectric system and the load during the piezo to EM charge transfer). However, the experimental value of load for which the assumption is not fulfilled, corresponding to $60 k \Omega$, is anyway lower than the typical input impedance of wireless autonomous systems, particularly when they operate in sleep mode (which corresponds to the case where devices operate most of the time at the largest extent). Recalling the expression of the critical load as:

$$
R_{C}=\frac{4}{C_{0} f_{0}\left(\eta_{\text {sece }}+\eta_{\text {em }} \frac{\beta^{2}}{L_{0}} \frac{C_{0}}{\alpha^{2}}\right)}
$$

and substituting the experimental values of the involved parameters (considering the additional capacitor of $0.22 \mu \mathrm{F}$ in parallel to the piezoelectric system), a value of $73 \mathrm{k} \Omega$ of theoretical critical load is obtained, thus quite close to the experimental one. Apart from the values of harvested powers within the "direct connection between piezo and load" area, slight discrepancies between the theoretical predictions and the experimental results are mainly due to diodes losses (when considering lower values of load resistors) and to storage capacitors losses (when considering high values of load resistors - higher than $10^{6} \Omega$ ).

In addition, the equivalent maximum harvested and extracted powers that can be achieved with the SECE case, respectively corresponding to $313 \mu \mathrm{W}$ and $455 \mu \mathrm{W}$, have been theoretically evaluated based on the identifications of the system parameters. Hence, in this case, the power gains of the proposed SECICE technique w.r.t. the SECE one correspond to 1.5 and 1.8 for the extracted and harvested powers, respectively, confirming the theoretical predictions.

Nevertheless, in realistic applications, extracting electrical energy from the electromechanical structure leads to a damping effect, thus limiting the harvested power to a value defined by:

$$
P_{\text {lim }}=\frac{F_{m}^{2}}{8 C}
$$

Hence, conducting the analysis as a function of a constant vibration magnitude is no longer valid, as the energy scavenging process leads to a reduction of the displacement magnitude. Consequently, it is next considered that the structure is vibrating at the resonance frequency and driven by a constant sinusoidal acceleration of $4.2 \mathrm{~ms}^{-2}(0.43$ g). In addition, the coupling coefficient of the piezoelectric system, which presents the highest coupling, is artificially decreased by adding capacitors in parallel to the 
Theoretical Extracted/Harvested Powers

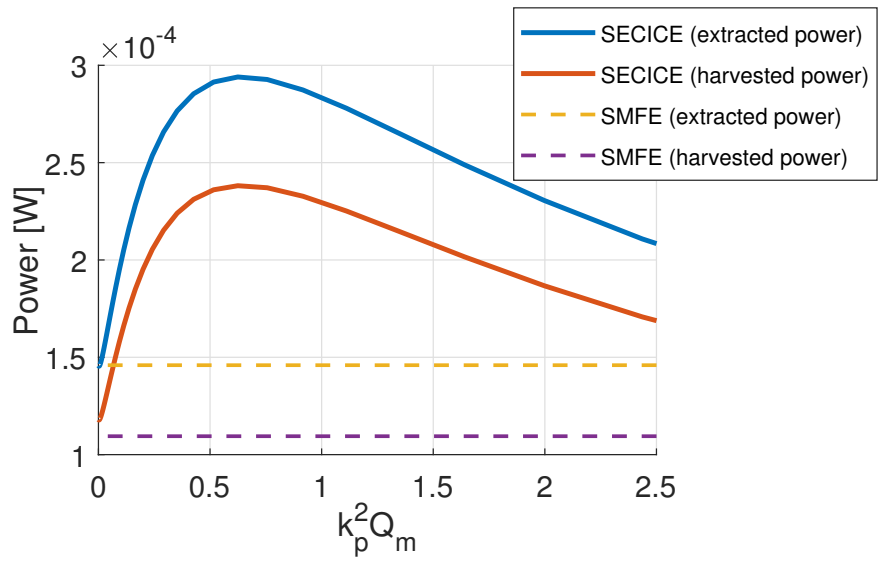

Experimental Extracted/Harvested Powers

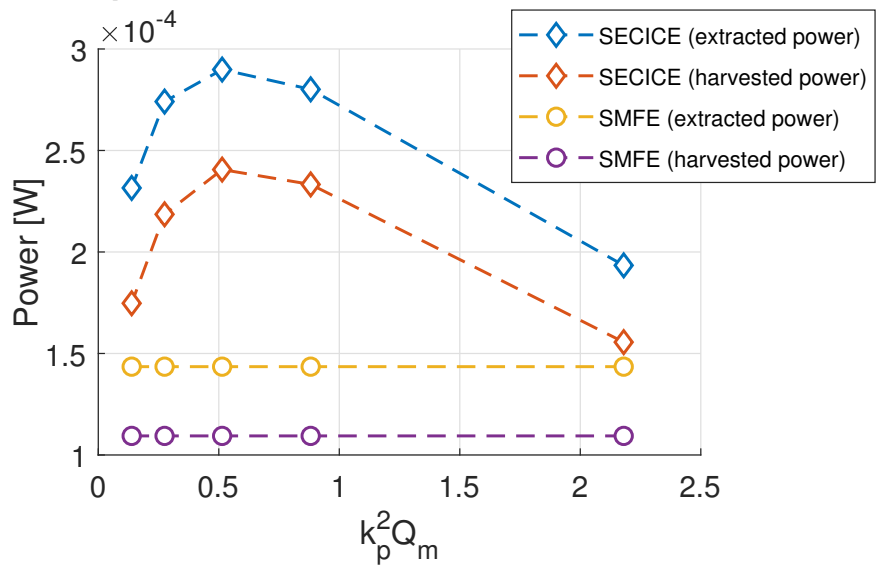

Figure 15: Experimental and theoretical harvested and extracted powers in the constant acceleration case

piezoelectric element. Experimental results in this case are depicted in figure 15, plotting the harvested and extracted powers (w.r.t. the power limit, as per equation (41)) versus the piezoelectric FoM $k_{p}{ }^{2} Q_{m}$. As predicted, the SECICE technique allows harvesting more energy than the SMFE interface, especially in the region of low coupled/highly damped structures, consistent with actual applications of small-scale energy harvesting systems [1]. In the considered case and coupling ranges, it is noteworthy that the SECICE always outperforms the SMFE, mainly due to the rather low coupling of the electromagnetic structure.

Finally, as previously conducted, when theoretically comparing the obtained results with the SECE technique, the use of SECICE interface over the SECE one is beneficial for values of coupling lower than $k_{p}{ }^{2} Q_{m}=0.5$ (reflecting existing applications of smallscale piezoelectric energy harvesting systems [19]), as theoretically discussed. 


\section{Conclusion}

With in mind the objective of using as much as electroactive parts as possible on the same electrical circuit, the aim of this paper was to expose a nonlinear electronic interface dedicated to hybrid electromagnetic-piezoelectric systems with a focus on the enhancement of the output power of the electromagnetic element addressing the issue of impedance matching. The interface has been derived from a combination of the previously developed SECE and SMFE schemes, respectively developed for piezo and EM transductions. Similarly to the original interfaces, the proposed SECICE interface is relatively independent of the load as well (except when there is a direct connection of the piezoelectric element to the load, which happens at relatively low rectified voltage/load values). More particularly, the technique demonstrated how the charge transfer from the piezoelectric element to the electromagnetic system leads to an extracted power gain of 6 and 4 with respect to the pure resistive EM (i.e. electromagnetic system connected to a varying load) and SMFE techniques, respectively. This power gain is achievable when considering low coupled electroactive systems, as for these systems the energy transfer process does not impact the overall dynamics of the transducers. Further works can be addressed to make the electronic system self-powered, controlling the switches directly with the signals from the transducers involved. In this regard, a self-powered harvesting integrated circuit that includes self-tuning capabilities was recently developed, addressing also the issue of frequency bandwidth [25]. Moreover, as in realistic applications the piezoelectric and electromagnetic transducers may vibrate separately (being composed by different movable components), the investigation of the effect of subjecting the transducers to different vibrations could be envisaged.

\section{Acknowledgments}

The authors gratefully acknowledge the financial support from European Union's Horizon 2020 Research and Innovation Programme under the Marie Skłodowska-Curie Grant Agreement No. 722496.

\section{References}

[1] Emmanuelle Arroyo and Adrien Badel. Electromagnetic vibration energy harvesting device optimization by synchronous energy extraction. Sensors and Actuators A: Physical, 171(2):266$273,2011$.

[2] Anthony Barré, Benjamin Deguilhem, Sébastien Grolleau, Mathias Gérard, Frédéric Suard, and Delphine Riu. A review on lithium-ion battery ageing mechanisms and estimations for automotive applications. Journal of Power Sources, 241:680-689, 2013.

[3] Stephen P Beeby and Terence O'Donnell. Electromagnetic energy harvesting. In Energy Harvesting Technologies, pages 129-161. Springer, 2009.

[4] Alexis Brenes, Elie Lefeuvre, Seonho Seok, and Chan-Sei Yoo. Tunable unipolar synchronized electric charge extraction strategy for piezoelectric energy harvesting. Journal of Intelligent Material Systems and Structures, 30(11):1629-1638, 2019. 
[5] Pedro Carneiro, Marco P Soares dos Santos, André Rodrigues, Jorge AF Ferreira, José AO Simões, A Torres Marques, and Andrei L Kholkin. Electromagnetic energy harvesting using magnetic levitation architectures: A review. Applied Energy, 260:114191, 2020.

[6] Xuning Feng, Minggao Ouyang, Xiang Liu, Languang Lu, Yong Xia, and Xiangming He. Thermal runaway mechanism of lithium ion battery for electric vehicles: A review. Energy Storage Materials, 10:246-267, 2018.

[7] David Gibus, Pierre Gasnier, Adrien Morel, Fabien Formosa, Ludovic Charleux, Sébastien Boisseau, Gaël Pillonnet, Carlos Augusto Berlitz, Anthony Quelen, and Adrien Badel. Strongly coupled piezoelectric cantilevers for broadband vibration energy harvesting. Applied Energy, 277:115518, 2020.

[8] Jayavardhana Gubbi, Rajkumar Buyya, Slaven Marusic, and Marimuthu Palaniswami. Internet of things (iot): A vision, architectural elements, and future directions. Future generation computer systems, 29(7):1645-1660, 2013.

[9] Daniel Guyomar, Adrien Badel, Elie Lefeuvre, and Claude Richard. Toward energy harvesting using active materials and conversion improvement by nonlinear processing. IEEE transactions on ultrasonics, ferroelectrics, and frequency control, 52(4):584-595, 2005.

[10] Daniel Guyomar and Mickaël Lallart. Recent progress in piezoelectric conversion and energy harvesting using nonlinear electronic interfaces and issues in small scale implementation. Micromachines, 2(2):274-294, 2011.

[11] Daniel Guyomar, Gaël Sebald, Sébastien Pruvost, Mickaël Lallart, Akram Khodayari, and Claude Richard. Energy harvesting from ambient vibrations and heat. Journal of Intelligent Material Systems and Structures, 20(5):609-624, 2009.

[12] Yushin Hara, Kensuke Saito, and Kanjuro Makihara. Compact, digital and self-powered piezoelectric vibration energy harvester with generation control using voltage measurement circuit. Sensors and Actuators A: Physical, 299:111609, 2019.

[13] Thomas Huguet, Adrien Badel, Olivier Druet, and Mickaël Lallart. Drastic bandwidth enhancement of bistable energy harvesters: Study of subharmonic behaviors and their stability robustness. Applied energy, 226:607-617, 2018.

[14] Thomas Huguet, Adrien Badel, and Mickaël Lallart. Exploiting bistable oscillator subharmonics for magnified broadband vibration energy harvesting. Applied Physics Letters, 111(17):173905, 2017.

[15] Mickaël Lallart. Amélioration de la conversion électroactive de matériaux piézoélectriques et pyroélectriques pour le contrôle vibratoire et la récupération d'énergie-Application au contrôle de santé structurale auto-alimenté. PhD thesis, 2008.

[16] Mickaël Lallart and Daniel J Inman. Mechanical effect of combined piezoelectric and electromagnetic energy harvesting. In Structural Dynamics and Renewable Energy, Volume 1, pages 261-272. Springer, 2011.

[17] Mickaël Lallart and Giulia Lombardi. Synchronized switch harvesting on electromagnetic system: a nonlinear technique for hybrid energy harvesting based on active inductance. Energy Conversion and Management, 203:112135, 2020.

[18] Mickaël Lallart, Christophe Magnet, Claude Richard, Elie Lefeuvre, Lionel Petit, Daniel Guyomar, and Frédéric Bouillault. New synchronized switch damping methods using dual transformations. Sensors and Actuators A: Physical, 143(2):302-314, 2008.

[19] Mickaël Lallart, Wen-Jong Wu, Yuchieh Hsieh, and Linjuan Yan. Synchronous inversion and charge extraction (sice): a hybrid switching interface for efficient vibrational energy harvesting. Smart Materials and Structures, 26(11):115012, 2017.

[20] E Lefeuvre, A Badel, A Brenes, S Seok, M Woytasik, and CS Yoo. Analysis of piezoelectric energy harvesting system with tunable sece interface. Smart Materials and Structures, 26(3):035065, 2017.

[21] Elie Lefeuvre, Adrien Badel, Claude Richard, and Daniel Guyomar. Piezoelectric energy harvesting device optimization by synchronous electric charge extraction. Journal of Intelligent Material 
Systems and Structures, 16(10):865-876, 2005.

[22] Elie Lefeuvre, Adrien Badel, Claude Richard, Lionel Petit, and Daniel Guyomar. A comparison between several vibration-powered piezoelectric generators for standalone systems. Sensors and Actuators A: Physical, 126(2):405-416, 2006.

[23] Haipeng Liu, Shiqiao Gao, Junru Wu, and Ping Li. Study on the output performance of a nonlinear hybrid piezoelectric-electromagnetic harvester under harmonic excitation. Acoustics, 1(2):382-392, Apr 2019.

[24] Huicong Liu, Hailing Fu, Lining Sun, Chengkuo Lee, and Eric M Yeatman. Hybrid energy harvesting technology: From materials, structural design, system integration to applications. Renewable and Sustainable Energy Reviews, page 110473, 2020.

[25] Adrien Morel, Gaël Pillonnet, Pierre Gasnier, Elie Lefeuvre, and Adrien Badel. Frequency tuning of piezoelectric energy harvesters thanks to a short-circuit synchronous electric charge extraction. Smart Materials and Structures, 28(2):025009, 2018.

[26] Joseph A Paradiso and Thad Starner. Energy scavenging for mobile and wireless electronics. IEEE Pervasive computing, (1):18-27, 2005.

[27] Shashank Priya, Hyun-Cheol Song, Yuan Zhou, Ronnie Varghese, Anuj Chopra, Sang-Gook Kim, Isaku Kanno, Liao Wu, Dong Sam Ha, Jungho Ryu, et al. A review on piezoelectric energy harvesting: materials, methods, and circuits. Energy Harvesting and Systems, 4(1):3-39, 2019.

[28] M Rajarathinam and SF Ali. Energy generation in a hybrid harvester under harmonic excitation. Energy Conversion and Management, 155:10-19, 2018.

[29] Mohsen Safaei, Henry A Sodano, and Steven R Anton. A review of energy harvesting using piezoelectric materials: state-of-the-art a decade later (2008-2018). Smart Materials and Structures, 28(11):113001, 2019.

[30] Faisal Karim Shaikh and Sherali Zeadally. Energy harvesting in wireless sensor networks: A comprehensive review. Renewable and Sustainable Energy Reviews, 55:1041-1054, 2016.

[31] Lihua Tang, Yaowen Yang, and Chee Kiong Soh. Toward broadband vibration-based energy harvesting. Journal of intelligent material systems and structures, 21(18):1867-1897, 2010.

[32] Sara Zolfaghar Tehrani, Hossein Ranjbar, Peter Vial, and Prashan Premaratne. A new efficient power management interface for hybrid electromagnetic-piezoelectric energy harvesting system. In 16th International Conference on Information Technology-New Generations (ITNG 2019), pages 537-542. Springer, 2019.

[33] RM Toyabur, M Salauddin, Hyunok Cho, and Jae Y Park. A multimodal hybrid energy harvester based on piezoelectric-electromagnetic mechanisms for low-frequency ambient vibrations. Energy Conversion and Management, 168:454-466, 2018.

[34] T Wacharasindhu and JW Kwon. A micromachined energy harvester from a keyboard using combined electromagnetic and piezoelectric conversion. Journal of Micromechanics and Microengineering, 18(10):104016, 2008.

[35] Chongfeng Wei and Xingjian Jing. A comprehensive review on vibration energy harvesting: Modelling and realization. Renewable and Sustainable Energy Reviews, 74:1-18, 2017.

[36] CB Williams and Rob B Yates. Analysis of a micro-electric generator for microsystems. sensors and actuators A: Physical, 52(1-3):8-11, 1996.

[37] Huakang Xia, Renwen Chen, and Long Ren. Analysis of piezoelectric-electromagnetic hybrid vibration energy harvester under different electrical boundary conditions. Sensors and Actuators A: Physical, 234:87-98, 2015.

[38] Hong-Xiang Zou, Lin-Chuan Zhao, Qiu-Hua Gao, Lei Zuo, Feng-Rui Liu, Ting Tan, Ke-Xiang Wei, and Wen-Ming Zhang. Mechanical modulations for enhancing energy harvesting: Principles, methods and applications. Applied Energy, 255:113871, 2019. 